\title{
O Desenvolvimento do Comportamento do Bebê Prematuro no Primeiro Ano de Vida
}

\author{
Behavior Development of Preterm Infants in the First Year of Life
}

\author{
Elizabeth Batista Pinto* \\ Universidade de São Paulo, Brasil \& Roosevelt Academy, Holanda
}

\begin{abstract}
Resumo
Este artigo apresenta uma investigação do comportamento de 21 bebês prematuros (de 33 a 36 semanas de gestação), de 1 a 12 meses, realizada no Hospital da Universidade de São Paulo, Brasil, utilizando a Escala de Desenvolvimento do Comportamento da Criança - EDCC. Os resultados mostraram que os prematuros, a partir do $3^{\circ}$ mês apresentaram uma recuperação importante no rítmo de desenvolvimento do comportamento, reduzindo de modo estatisticamente significante a frequência de comportamentos com indicadores patológicos, mas mantendo um fator de risco de $23 \%$ de comportamentos não-normalizados aos 12 meses incompletos. Este estudo contribui com elementos que podem favorecer o acompanhamento do processo de desenvolvimento do comportamento destas crianças e na detecção precoce de atrasos ou possíveis distúrbios neste processo.

Palavras-chave: Desenvolvimento; comportamento; escala; bebê; prematuridade.
\end{abstract}

\begin{abstract}
This article presents a research about the behavior development of 21 preterm babies (between 33 and 36 weeks of pregnancy), from 1 to 12 months old, at the Hospital of the University of São Paulo, in Brazil, using the Child Behavior Development Scale - CBDS. The results showed that the preterm infants had an important improvement in the behavior development rhythm after the $3^{\text {rd }}$ month of age, reducing in a statistically significant way the behaviors with pathological signs, but maintaining risk factors in $23 \%$ of non-normalized behaviors at 12 months of age. This study provides elements that can help the follow-up of the behavior development process of these children and the early detection of delays or possible disorders in that process.

Keywords: Development; behavior; scale; baby; infant; preterm.
\end{abstract}

O avanço científico na área da saúde nos últimos anos vem ampliando as perspectivas dos programas de prevenção dirigidos à criança e à família. Os especialistas que trabalham nesse campo têm grandes preocupações com relação ao desenvolvimento da criança no primeiro ano de vida, tendo em vista as alterações que podem ocorrer em função de alto risco, prematuridade, distúrbios genéticos ou neurológicos, deficiências sensoriais, distúrbios afetivos ou outros (Atkin, Supervielle, Sawyer, \& Cantón, 1987; Coll, 1990; Ortiz, 1987; Victora, Barros, Martines, Béria, \& Vaughan, 1985; Widerstrom, 1999).

A prematuridade é estabelecida a partir de parâmetros colocados em evidência pelos exames médicos (dados clínicos e ultrasonográficos) e pela avaliação anatômica da criança (principalmente peso e estatura). A definição de prematuridade da Organização Mundial de Saúde ([WHO], 1980) que toma como ponto de partida a idade gestacional, estabelece que é prematura a criança nascida de uma ges-

\footnotetext{
* Endereço para correspondência: Noorwegenlaan 9 , Brouwershaven, Holanda, 4318 CB.E-mail: ebatista@usp.br ou e.wiese@roac.nl

Apoio: Coordenação de Aperfeiçoamento de Pessoal de Nível Superior (CAPES)/Programa de Apoio à Educação Especial (PROESP).
}

tação com tempo inferior a 37 semanas, contadas a partir da última menstruação. O bebê nascido entre 32 e 35 semanas de gestação é considerado como uma criança $d e$ risco e o bebê nascido antes de 32 semanas é considerado de alto risco. A definição, segundo os critérios relativos ao peso estabelece como prematura a criança que nasceu antes do final da gestação e com um peso inferior a $2.500 \mathrm{~g}$.

Também é importante a associação entre a idade gestacional e o peso da criança, pois uma criança hipotrófica - de baixo peso quando considerada a idade gestacional - pode apresentar déficits mais importantes do que um prematuro eutrófico - com peso apropriado para sua idade gestacional.

Os bebês prematuros constituem, em média, 5,6\% dos nascimentos no Brasil, segundo a taxa de prematuridade do Sistema Único de Saúde - SUS (citado pela Agência Nacional de Saúde Suplementar [ANS], 2005). As causas da interrupção precoce da gravidez e de um parto prematuro podem ser diversas, podendo estar implicados fatores relativos à saúde da mãe e/ou do bebê.

A evolução da Neonatologia, a partir da década de 70, que vem reduzindo a mortalidade de bebês nascidos prematuros, tem como desafio a questão da morbidade e da qualidade de vida dessas crianças (Lequien, 1999). O nascimento prematuro constitui um importante fator de vulne- 
rabilidade, que pode comprometer tanto a saúde e como o desenvolvimento da criança (Batista Pinto, 2000; François, Battisti, Bertrand, Kalenga, \& Langhendries, 1998; Guralnick, 1998; Mellier, 1999).

A prevalência de distúrbios do desenvolvimento na prematuridade está associada a diversos parâmetros como a idade gestacional, o peso ao nascimento e as dificuldades médicas que podem ocorrer principalmente no período neonatal (sobretudo distúrbios respiratórios, digestivos e neurológicos).

Para Mellier (1999), a prematuridade constitui uma situação excepcional tanto para a criança como para seus pais, pois implica no aumento de diversos fatores de vulnerabilidade frente aos distúrbios de desenvolvimento, que devem ser compreendidos a partir de uma múltipla influência: biológica, ambiental e social.

O’Brien, Soliday e McCluskey-Fawcett (1995) também referiram que fatores biológicos e psicosociais atuam conjuntamente atenuando ou agravando os efeitos da prematuridade no desenvolvimento da criança. Já Widerstrom (1999), enfatizou a importância do fator social, pois mães economicamente carentes ou que abusam de drogas ou de álcool, assim como as mães adolescentes, apresentam maior risco de dar à luz a uma criança prematura.

No Brasil, Linhares, Carvalho, Bordin e Jorge (1999) referiram que fatores sociais associados à condição de pobreza e suas adversidades podem agravar os riscos relacionados à prematuridade, prejudicando o desenvolvimento da criança prematura mas, por outro lado, fatores ambientais protetores combinados podem favorecer a resiliência e contribuir positivamente no processo de desenvolvimento do comportamento da criança.

Além dos fatores relativos à saúde física e às condições sociais, pode-se também considerar a relevância de fatores emocionais, pois em estudos realizados em psicoterapia pais/ bebê prematuro (Batista Pinto, 2000), constatou-se elevada frequência de estresse emocional e de dificuldades afetivas durante a gestação, tais como abandono do companheiro, morte de pais ou parentes próximos, gravidez indesejada fora do casamento e outras, conforme o relato das mães.

Mais ainda, conforme Barratt, Roach e Leavitt (1996) observaram, as mães de crianças prematuras com mais de vinte meses de idade podem ter seus comportamentos afetados por circunstâncias associadas ao nascimento prematuro, mesmo quando a criança apresenta um desenvolvimento cognitivo e de linguagem adequado para a idade.

Mellier (1999) realizou um levantamento das pesquisas sobre os principais distúrbios associados à prematuridade, enfatizando a importância das diversas formas de avaliação precoce e medidas preventivas com o objetivo de prevenir algumas consequiências negativas da prematuridade e do estresse parental resultante da hospitalização sobre o desenvolvimento da criança.

Pode-se, portanto, concluir que o termo prematuro não se refere a uma população homogênea de crianças. Em função disso, Dalla Piazza (1997) salientou a importância de se especificar qual a população de prematuros estudada, a fim de se assegurar conclusões mais especificas.
Importante salientar que um período prolongado em ambiente hospitalar e os procedimentos médicos aos quais a criança prematura pode ter sido submetida no início da vida, também podem influir negativamente sobre as suas integrações sensoriais e afetar o desenvolvimento do seu comportamento, inclusive a sua estruturação cognitiva. Portanto, o nascimento prematuro e a situação de hospitalização que, em geral, se segue, podem criar um estresse emocional, que pode afetar a emergência de competências, tanto na criança como nos pais (Padovani, Linhares, Carvalho, Duarte, \& Martinez, 2004).

Sabe-se que o processo de desenvolvimento nos primeiros meses apresenta toda uma série de manifestações somáticas e comportamentais, devendo a criança regular os ritmos dos estados de: vigília/sono, fome/saciedade, atenção/habituação, atividade/repouso. Os parâmetros de desenvolvimento modificam-se com a idade, mas faz-se importante considerar as influências do ambiente e das características individuais da criança (Batista Pinto, Vilanova, \& Vieira, 1997).

Dentre as muitas questões relativas à prematuridade, os psicólogos têm-se interessado em pesquisar principalmente o desenvolvimento da criança e suas competências cognitivas e sociais, buscando compreender e descrever o impacto da prematuridade e eventualmente definir práticas terapêuticas preventivas para os possíveis distúrbios do desenvolvimento do comportamento.

Diversos estudos têm mostrado o recém-nascido prematuro como um parceiro capaz e ativo em sua interação com o ambiente (Bullinger \& Goubet, 1999; Thoman \& Ingersoll, 1993; Thoman, Ingersoll, \& Acebo, 1991; Tremblay-Leveau, Lemaitre-Boquet, Megan, Renard, \& Delaunay, 1999). Outros apontaram que a prematuridade pode afetar condutas precoces de orientação do bebê no ambiente, tanto em sua relação com pessoas como com objetos, assim como a regulação dos estados de atenção e de familiarização diante de eventos perceptivos repetidos, sugerindo que a criança prematura não apresenta na interação as mesmas competências da criança nascida a termo, descrevendo a prematura como menos sensível e menos reativa aos estímulos externos (Als, 1995; Charavel, 2000).

Ramos (1999) fez um estudo comparando o desenvolvimento do comportamento de crianças de seis a doze meses de idade, nascidas pré-termo e a termo, utilizando a Escala de Desenvolvimento do Comportamento da Criança EDCC (Batista Pinto et al., 1997), tendo encontrado diferenças estatisticamente significantes entre os dois grupos nos comportamentos classificados como Comportamento Axial Espontâneo Não-Comunicativo (tais como: puxa para sentar-se, mantém-se sentada com o apoio das mãos, arrasta-se, senta-se sem o apoio das mãos, mantém-se em pé com o mínimo apoio, engatinha, passa de prono para a posição sentada, caminha com auxílio, dá alguns passos sem apoio) com desenvolvimento mais lento nas crianças prematuras do sexo feminino e no conjunto das crianças prematuras (ambos os sexos considerados conjuntamente).

Linhares, Carvalho, Machado e Martinez (2003) investigaram o desenvolvimento do comportamento de 42 bebês 
prematuros, de menos de 34 semanas de idade gestacional e peso ao nascer inferior a $1500 \mathrm{~g}$, aos 6 e 11 meses, utilizando também a EDCC (Batista Pinto et al., 1997). Concluíram que o desempenho das crianças na $E D C C$ foi bom ou excelente, considerando a idade corrigida, verificando, no entanto, $30 \%$ de crianças com sinais de risco ou atraso no desenvolvimento do comportamento aos 6 meses de idade corrigida. Já aos 11 meses de idade corrigida as crianças mostraram um desenvolvimento do comportamento bom ( $\left.{ }^{3} 64 \%\right)$, com exceção dos comportamentos classificados como Axial Espontaneo Não-Comunicativo e Axial Espontaneo Comunicativo, que obtiveram um risco ou atraso respectivamente de $39 \%$ e $33 \%$.

Pérez-Ramos, Pérez-Ramos, Sá, Morais e Jaehn (1992) analisaram as principais técnicas de detecção, diagnóstico e intervenção frente aos distúrbios de desenvolvimento do comportamento da criança, sugerindo que os programas de intervenção deveriam ser iniciados o mais cedo possível para possibilitarem efeitos positivos duradouros.

O processo de desenvolvimento do comportamento da criança está associado à sua carga genética, às suas condições de saúde e às multiplas e complexas influências familiares, ambientais, culturais e sociais que a envolvem. Tal processo merece ser estudado em específico e em detalhe, em suas várias fases e características, sendo que o mesmo na criança prematura torna-se ainda mais complexo e inclui riscos adicionais no desenvolvimento do comportamento da criança.

Assim, a investigação descrita no presente artigo estudou detalhadamente o desenvolvimento do comportamento da criança nascida prematura, no primeiro ano de vida. Esta investigação, realizada em um programa de seguimento do desenvolvimento de bebês prematuros do Hospital da Universidade de São Paulo (HU/USP), incluiu sessões de atendimento mensal aos pais e ao bebê, nas quais, além da avaliação do desenvolvimento do comportamento, eram abordados temas variados relativos ao contexto familiar e ao desenvolvimento do bebê, identificadas dificuldades e eventuais problemas e distúrbios e fornecidas orientações para a promoção do desenvolvimento e para o favorecimento da interação pais/bebê.

\section{Objetivos}

O objetivo desta pesquisa foi estudar o desenvolvimento do comportamento de crianças de 1 a 12 meses incompletos de idade, de ambos os sexos, nascidas prematuras entre 33 e 36 semanas de gestação.

\section{Método}

\section{Participantes}

As crianças foram selecionadas, considerando os dados obtidos através de uma ficha de anamnese, dentre as nascidas prematuramente no HU/USP. O projeto de pesquisa foi aprovado pelo Comitê de Ética do HU/USP.

A amostra foi formada por 21 bebês de ambos os sexos, nascidos pré-termo entre 33 e 36 semanas de gestação e sem seqüelas neurológicas. Escolheu-se estudar este grupo de crianças prematuras, pois estas, apesar de apresentarem riscos no desenvolvimento do comportamento, desenvolvem, em geral, boa saúde física, sem as sequelas neurológicas, os distúrbios sensoriais ou outras patologias que com maior freqüência estão presentes em crianças prematuras de alto risco.

Apesar da proposta inicial ter sido de acompanhar o mesmo grupo de crianças longitudionalmente, mês a mês, ao longo do primeiro ano de vida, na pratica nem sempre isto foi possível, em função de desistências ou impossibilidades da família de trazer a criança mensalmente para a avaliação. Assim, optamos por fazer a análise das avaliações conjuntas de cada mês de idade, incluindo tanto os resultados de crianças que realizaram uma única avaliação em determinado mês como aquelas que prosseguiram em seguimento longitudinal nos meses seguintes.

Levando em conta as modificações no desenvolvimento do comportamento que podem ser observadas no decorrer do primeiro ano de vida, nesta investigação subdividiu-se a faixa etária estudada trimestralmente - de 1 a 3 meses, de 4 a 6 meses, de 7 a 9 meses e de 10 a 12 meses incompletos de idade - tendo excluído o período neonatal (de 1 a 29 dias de vida). Para fins de seleção da amostra considerou-se a idade real das crianças (em meses). Nas análises dos resultados além da idade real foi também considerada a idade corrigida.

\section{Procedimentos}

Para a avaliação do desenvolvimento do comportamento da criança escolheu-se a Escala de Desenvolvimento do Comportamento da Criança - EDCC (Batista Pinto et al., 1997). A $E D C C$ é um instrumento de observação interativa, de fácil aplicação e avaliação, que foi especialmente estruturado para a avaliação do desenvolvimento do comportamento de crianças de um a doze meses, que considera os comportamentos mais significativos nesta faixa etária e fornece uma indicação do rítmo e uma avaliação qualitativa do processo de desenvolvimento do comportamento da criança. Outro fator determinante em nossa escolha é o fato da $E D C C$ ter uma padronização cuidadosa, realizada com crianças brasileiras sadias, nascidas a termo e sem fatores de risco para o desenvolvimento do comportamento.

A EDCC analisa sessenta e quatro comportamentos observados no primeiro ano de vida, classificando-os em comportamento motor - axial e apendicular, espontâneo e estimulado - e em comportamento atividade - não-comunicativo e comunicativo. Na EDCC os comportamentos são também analisados quanto ao tipo de ocorrência, o que permite uma indicação do seu ritmo de desenvolvimento e possibilita uma estimativa se um determinado comportamento apresenta um desenvolvimento conforme o esperado, isto é, está em aquisição ou em aparecimento, se o comportamento está normalizado (com frequência esperada de $67 \%$ a $89 \%$ da população da faixa etária) ou se é não-normalizado, ou seja, de risco, ou se o comportamento está estabilizado (com frequência esperada igual ou superior a $90 \%$ da população da faixa etária) ou não-estabilizado, isto é, apresenta atraso na sua aquisição. 
Wiese, E. B. P. (2009). O Desenvolvimento do Comportamento do Bebê Prematuro no Primeiro Ano de Vida.

$\mathrm{O}$ atendimento inicial das crianças da amostra incluiu uma entrevista semi-dirigida com os pais para a obtenção dos dados de anamnese e a assinatura do consentimento dos pais para a pesquisa. Seguiu-se a observação e avaliação do desenvolvimento do comportamento da criança com a EDCC, em situação interativa, na presença da mãe ou dos pais.

Assim, a mãe ou os pais participaram das avaliações do desenvolvimento do comportamento de seus bebês, sendo que os comportamentos positivos obtidos em cada avaliação, e que indicavam competências do bebê, eram apontados aos pais pela psicóloga durante a consulta. Na mesma sessão eram discutidos aspectos gerais da fase de desenvolvimento do comportamento na qual se encontrava o bebê ou outras questões relacionadas trazidas pelos pais.

Os resultados de cada avaliação da criança foram analisados separadamente e posteriormente agrupados de acordo com as variáveis abaixo especificadas. Na análise dos resultados gerais, investigou-se o ritmo de desenvolvimento do comportamento analisando-se os dados dos bebês considerando-se sub-grupos independentes quanto: ao sexo (masculino e feminino); à idade, em cada trimestre $\left(1^{\circ}, 2^{\circ} 3^{\circ}\right.$ e $4^{\circ}$ trimestres do primeiro ano de vida) e ao tipo de comportamento (axial/apendicular, espontâneo/estimulado e não-comunicativo/comunicativo). Seguindo o modelo da EDCC (Batista Pinto et al., 1997), adotou-se nas análises os critérios de aparecimento $(0 \leq$ $p<67 \%)$, normalização $(67 \% \leq p<90 \%)$ e estabilização $(p \geq 90 \%)$ dos comportamentos.

$\mathrm{Na}$ análise estatística os resultados obtidos nas avaliações das crianças prematuras da amostra foram comparados com os resultados esperados, em criancas nascidas a termo (de acordo com os resultados de normatização da $E D C C$ ), utilizando-se testes não paramétricos (Siegel, 1975) e considerando-se as distribuições das variáveis estudadas (sexo, idade e tipo de comportamento). Os testes adotados foram o Teste de MacNemar e o Teste Exato de Fisher. Em todos os testes fixou-se em 0,05 ou $5 \%$ o nível para a rejeição da hipótese de nulidade $(p \leq 0,005)$.

\section{Resultados}

Foram realizadas no total 112 avaliações do comportamento com crianças do sexo masculino (64\%) e 64 com crianças do sexo feminino (36\%), totalizando 176 avaliações - 21 no primeiro trimestre (I), 54 no segundo trimestre (II), 51 no terceiro trimestre (III) e 50 no quarto trimestre (IV) do primeiro ano de vida.

\section{Amostra}

Para melhor caracterizar a amostra da população estudada, apresenta-se na Tabela 1 a média e o desvio padrão em algumas das suas principais características, considerando o foco da investigação.

$\mathrm{Na}$ Tabela 2 apresenta-se outras características familiares e gestacionais importantes da amostra da população estudada.
Tabela 1

Caracterização da Amostra quanto às Variáveis Fundamentais

\begin{tabular}{lc}
\hline Variáveis consideradas & Média (DP) \\
\hline Idade materna em anos & $26,50(6,46)$ \\
Idade paterna em anos & $29,73(6,10)$ \\
Idade gestacional em semanas & $35,00(0)$ \\
Peso ao nascer em gramas & $2226,11(717,44)$ \\
Estatura ao nascer em centímetros & $44,54(3,38)$ \\
\hline
\end{tabular}

Tabela 2

Caracterização da Amostra quanto à outras Variáveis Familiares e Gestacionais

\begin{tabular}{lc}
\hline Variáveis & $\begin{array}{c}\text { Frequência observada } \\
\text { (em porcentagem) }\end{array}$ \\
\hline Dos pais & \\
\hline Escolaridade dos pais & $30 \%$ Ensino Fundamental \\
& Incompleto \\
$34 \%$ Ensino Fundamental \\
$3 \%$ Ensino Médio Incompleto \\
$30 \%$ Ensino Médio \\
$3 \%$ sem informações
\end{tabular}

Da gestação

Pré-Natal

Antecedentes na Gestação*

Fatores Psicológicos**

Intercorrências

Primigesta

Parto

$89 \%$ Sim

$94 \%$ Sim

$89 \%$ Sim

$94 \%$ Sim

$44 \%$

$44 \%$ Natural

$56 \%$ Cesárea

\begin{tabular}{ll}
\hline Do neonato & \\
\hline Gêmeo & $22 \%$ \\
Apgar & $4 / 7 / 8$ a $9 / 10 / 10$ \\
RNPT - AIG & $67 \%$ \\
RNPT - PIG & $22 \%$ \\
RNPT - GIG & $11 \%$ \\
Ventilação & $89 \% \mathrm{Sim}$ \\
UTI & $56 \% \mathrm{Sim}$ \\
Anoxia & $22 \% \mathrm{Sim}$ \\
Icterícia & $78 \% \mathrm{Sim}$ \\
Amamentação ao seio & $83 \% \mathrm{Sim}$
\end{tabular}

Notas. Legenda: RNPT = recém-nascido pré-termo; AIG = adequado para a idade gestacional; PIG = pequeno para a idade gestacional; GIG = grande para a idade gestacional. * Antecedentes gestacionais de risco: bolsa rota, pressão alta, sífilis materna, diabetes gestacional, retardo no desenvolvimento intrauterino, placenta prévia, descolamento de placenta, hemorragia e artrite gonocócica materna. ** Fatores psicológicos de risco: gestação sem o apoio do pai da criança, depressão materna, problemas conjugais, problemas familiares.

Apresenta-se a seguir os resultados obtidos no desenvolvimento do comportamento das crianças prematuras de 1 a 
12 meses incompletos, em cada tipo de comportamento estudado, considerando o sexo e a idade em trimestres.

Quanto ao Sexo. A Figura 1 apresenta as freqüências relativas (em porcentagem) de comportamentos normalizados e estabilizados das crianças prematuras de 1 a 12 meses incompletos, considerando separadamente cada sexo e o total das crianças.

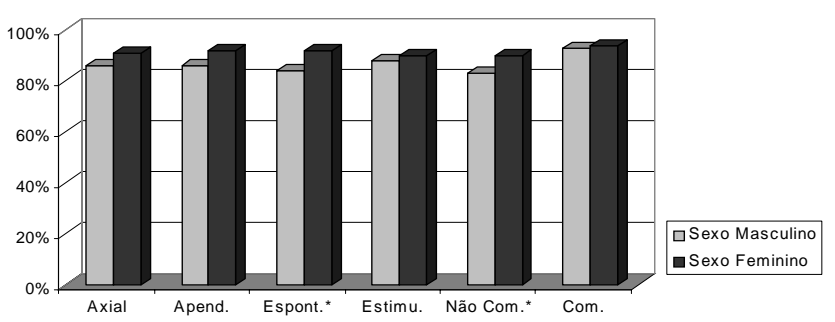

Figura 1. Distribuição das frequências relativas totais (\%) dos comportamentos normalizados e estabilizados de 1 a 12 meses incompletos, considerando o sexo

A frequiência relativa de comportamentos estabilizados no sexo masculino (67\%) e no feminino (74\%) é maior do que a de comportamentos normalizados. Este resultado indica que as crianças prematuras estudadas constituem, de modo geral, um grupo que apresenta riscos no desenvolvimento de seus comportamentos (33\% no sexo masculino e $26 \%$ no sexo feminino de comportamentos não normalizados), porém com uma porcentagem baixa de indicadores de possíveis distúrbios no desenvolvimento dos comportamentos (14\% no sexo masculino e $9 \%$ no sexo feminino de comportamentos não-estabilizados).

Observa-se que a maioria dos comportamentos está normalizada para ambos os sexos, na idade esperada. Apesar de, no total, o sexo feminino ter apresentado uma freqüência relativa de comportamentos normalizados e estabilizados (conforme o esperado para a idade) ligeiramente maior que o sexo masculino, a diferença não é estatisticamente significante.

As Figuras 2 e 3 apresentam, respectivamente, as frequiências relativas de comportamentos normalizados e estabilizados dos comportamentos axiais, apendiculares, espontâneos, estimulados, não comunicativos e comunicativos, das crianças prematuras de 1 a 12 meses incompletos, considerando o sexo.

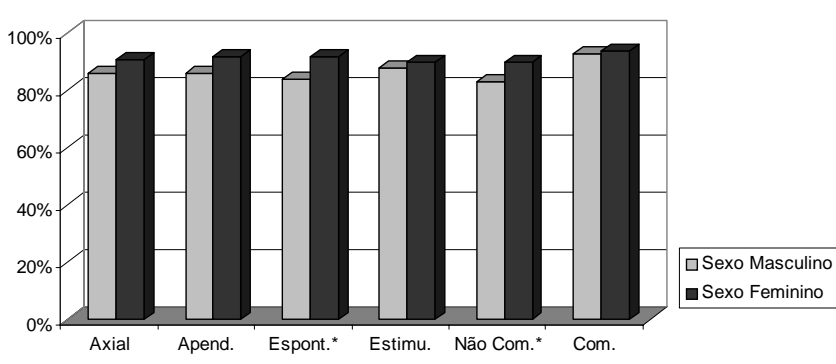

Figura 2. Distribuição das frequências relativas (\%) dos comportamentos normalizados de 1 a 12 meses, considerando o tipo de comportamento e o sexo

Nota. * Diferença estatisticamente significante entre os sexos.

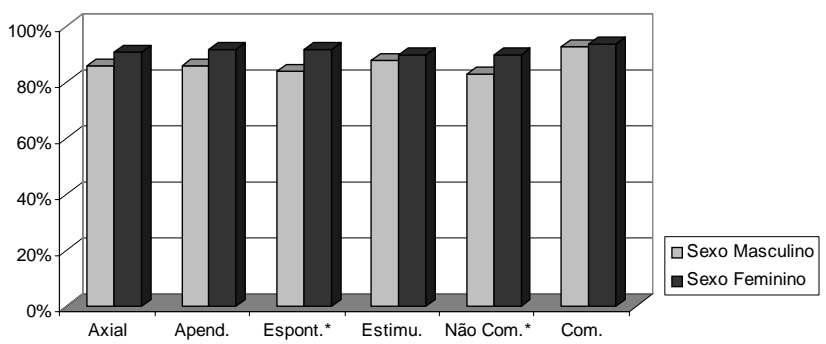

Figura 3. Distribuição das frequências relativas (\%) dos comportamentos estabilizados de 1 a 12 meses, considerando o tipo de comportamento e o sexo

Nota. * Diferença estatisticamente significante entre os sexos.

Quanto ao desenvolvimento dos comportamentos axiais, apendiculares, espontâneos, estimulados, não-comunicativos e comunicativos constatou-se que a frequêencia geral de comportamentos estabilizados foi maior do que a de comportamentos normalizados.

De forma geral o sexo feminino apresentou um desenvolvimento do comportamento em ritmo mais rápido do que o sexo masculino, com diferença estatisticamente significante nas freqüências de normalização dos comportamentos axiais e espontâneos, e de estabilização dos comportamentos espontâneos e não-comunicativos.

Quanto à Idade. A Figura 4 apresenta as freqüências relativas de comportamentos normalizados e estabilizados das crianças prematuras de ambos os sexos, considerando os quatro trimestres do primeiro ano de vida.

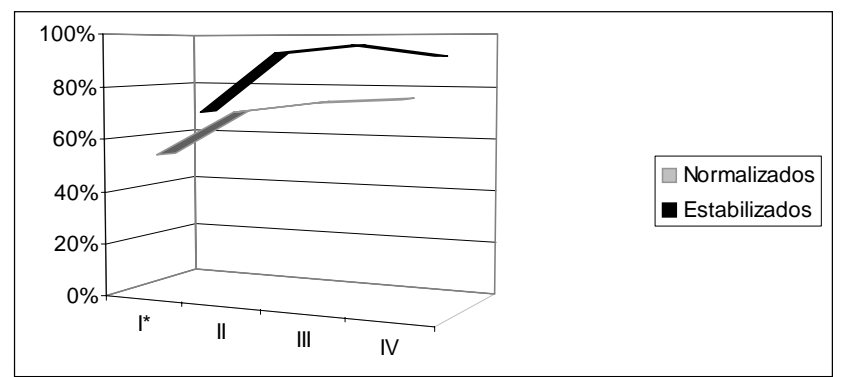

Figura 4. Distribuição das frequências relativas (\%) dos comportamentos normalizados e estabilizados considerando os quatro trimestres do primeiro ano de vida

Nota. * Diferença estatisticamente significante entre os trimestres.

Como se pode observar a frequiência total de comportamentos normalizados e estabilizados no primeiro trimestre (I) é bem inferior aos outros trimestres (II, III e IV), sendo a diferença estatisticamente significante $(p=0,005$ para a normalização e $p=0,000$ para a estabilização).

Assim, de forma geral, as crianças prematuras de ambos os sexos investigadas neste estudo demonstraram uma boa recuperação no rítmo de desenvolvimento do seu comportamento no primeiro ano a partir do $3^{\circ}$ mês de vida, reduzindo consideravelmente (e de forma estatisticamente significante) os comportamentos com indicadores patológicos. Este grupo mostrou também, no final do primeiro ano de vida, a freqüência relativa de $93 \%$ dos comportamentos 
Wiese, E. B. P. (2009). O Desenvolvimento do Comportamento do Bebê Prematuro no Primeiro Ano de Vida.

estabilizados na criança nascida a termo, apresentando portanto um total de apenas $7 \%$ de comportamentos não-estabilizados.

No entanto, quando considerados os comportamentos de risco (normalizados no período), obteve-se $77 \%$ dos comportamentos normalizados na criança a termo, indicando na criança prematura um fator de risco de $23 \%$ em comportamentos não-normalizados no desenvolvimento do comportamento aos 12 meses incompletos

Quanto ao desenvolvimento do comportamento da criança prematura ao longo dos quatro trimestres do primeiro ano de vida, constatou-se ainda que a freqüência de comportamentos estabilizados foi maior do que a de comportamentos normalizados nos diferentes tipos de comportamentos estudados (excetuando-se os comportamentos apendiculares no primeiro trimestre que apresentaram igual frequiência de normalização e de estabilização).
Nos resultados obtidos no primeiro trimestre (I), as crianças prematuras apresentaram um rítmo de desenvolvimento inferior aos outros trimestres (II, III e IV), com diferença estatisticamente significante na normalização dos comportamentos axiais $(p=0,043)$, espontâneos $(p=0,008) \mathrm{e}$ não-comunicativos $(p=0,001)$ e na estabilização dos comportamentos axiais $(p=0,001)$, apendiculares $(p=0,004)$, estimulados $(p=0,000)$ e não-comunicativos $(p=0,000)$. Observa-se, portanto, dificuldades mais acentuadas no desenvolvimento dos comportamentos no primeiro trimestre de vida, principalmente nos comportamentos axiais e nãocomunicativos.

Mesmo se considerada a idade corrigida, as crianças prematuras apresentaram atraso importante na aquisição da estabilização de diversos comportamentos, para ambos os sexos, como pode ser observado na Tabela 3.

Tabela 3

Relação dos Comportamentos com Atraso na Aquisição da Estabilização, em Crianças Prematuras de Ambos os Sexos, com Idade Corrigida

Tipo de comportamento

Axial Espontâneo Não Comunicativo

Axial Espontâneo Comunicativo

Axial Estimulado Não Comunicativo

Axial Estimulado Comunicativo

Apendicular Espontâneo Não Comunicativo

Apendicular Espontâneo Comunicativo Apendicular Estimulado Não Comunicativo

Apendicular Estimulado Comunicativo
Comportamento

6. Puxa para sentar-se

18. Emite sons vocalicos

19. Repete os próprios sons

21. Repete a mesma silaba

29. Sorri e vocaliza diante do espelho

38. Tem preensão palmar simples

42. Transfere objeto de uma mão para a outra

46. Tem preensão em pinça

50. Chocalha brinquedo

51. Tira pinos grandes

57. Responde a "vem" estendendo os braços
Apresenta-se a seguir nas Tabelas 4 e 5 uma proposta para a Escala de Desenvolvimento do Comportamento da Criança Prematura -EDCP, respectivamente para o sexo masculino e para o feminino.

\section{Discussão}

Considerando-se a vulnerabilidade do nascimento prematuro frente aos distúrbios de desenvolvimento, faz-se necessário o acompanhamento cuidadoso do desenvolvimento do comportamento da criança. A avaliação do desenvolvimento do comportamento através da Escala de Desenvolvimento do Comportamento da Criança - EDCC (Batista Pinto et al., 1997) pode favorecer a detecção precoce de atrasos e dificuldades no desenvolvimento do comportamento e contribuir para prevenir os efeitos prejudiciais da prematuridade na criança, seja no planejamento detalhado de estratégias de estimulação, no encaminhamento para especialistas ou no aprimoramento do conhecimento e da sensibilidade parental sobre o desenvolvimento da criança com uma possível redução dos distúrbios na interação pais/criança.

Os resultados deste estudo corroboram os de Thoman et al. (1991), Thoman e Ingersoll (1993), Bullinger e Goubet (1999) e Tremblay-Leveau et al. (1999), que apontam o prematuro como um parceiro ativo em sua interação com o ambiente. Mais além, este estudo mostrou um avanço com relação aos mencionados pois mostrou em detalhe cada comportamento apontando como a prematuridade, mesmo reduzida - como o nascimento após 33 a 36 semanas de gestação - pode afetar o comportamento do bebê em sua relação com o ambiente, tanto com pessoas como com objetos e a sua atenção para eventos perceptivos, sugerindo que a criança prematura não apresenta na interação as mesmas competências no primeiro ano de vida da criança nascida a termo, mesmo quando considerada a idade corrigida, mostrando um ritmo mais lento de desenvolvimento e, paralelamente, um atraso na aquisição, normalização e/ ou estabilização em diversos comportamentos. 
Tabela 4

Escala de Desenvolvimento do Comportamento da Criança Prematura no Primeiro Ano de Vida - EDCP. Sexo Masculino

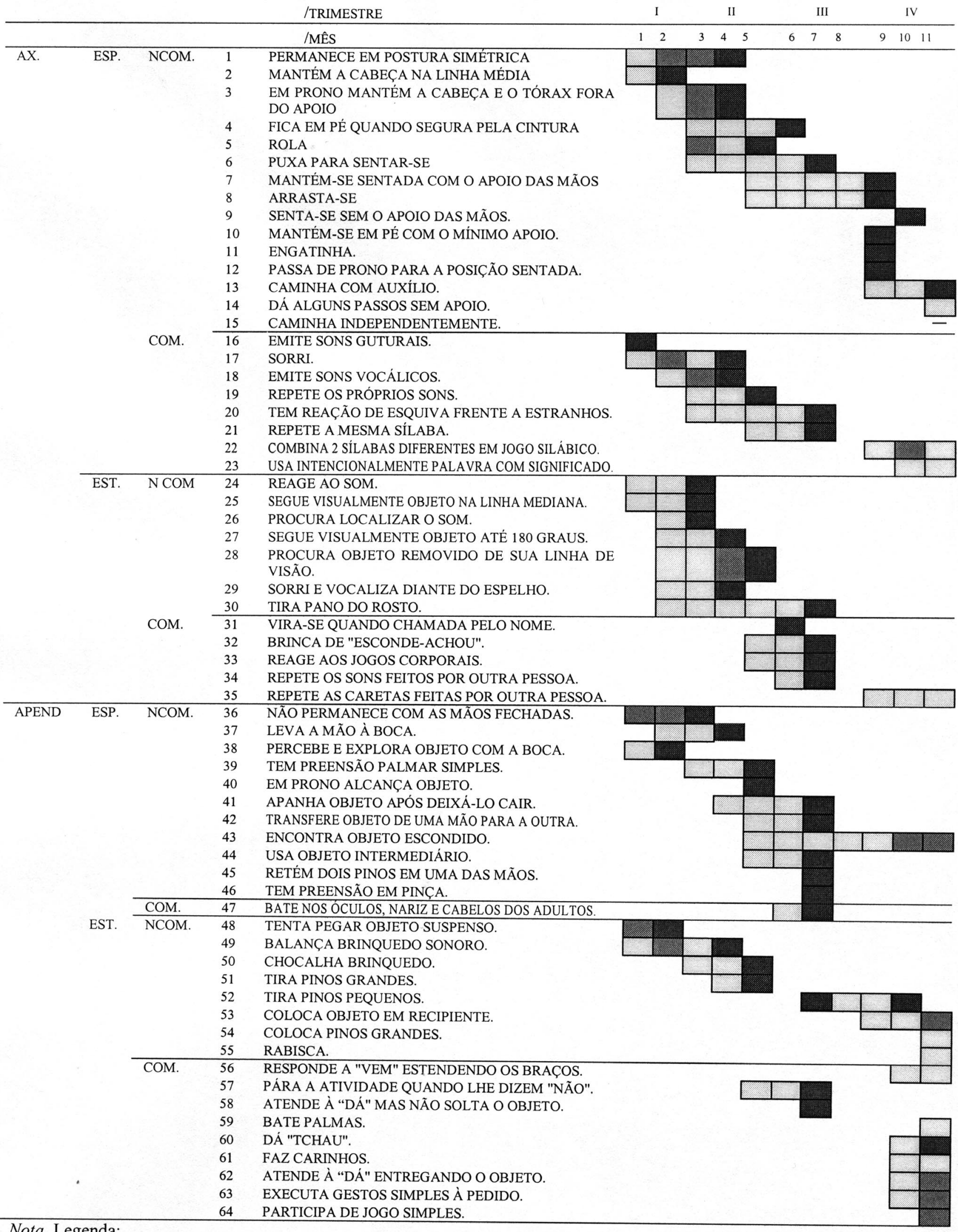

Nota. Legenda:

Aparecimento.

Normalização.

Estabilização. 
Wiese, E. B. P. (2009). O Desenvolvimento do Comportamento do Bebê Prematuro no Primeiro Ano de Vida.

Tabela 5

Escala de Desenvolvimento do Comportamento da Criança Prematura no Primeiro Ano de Vida - EDCP. Sexo Femini-

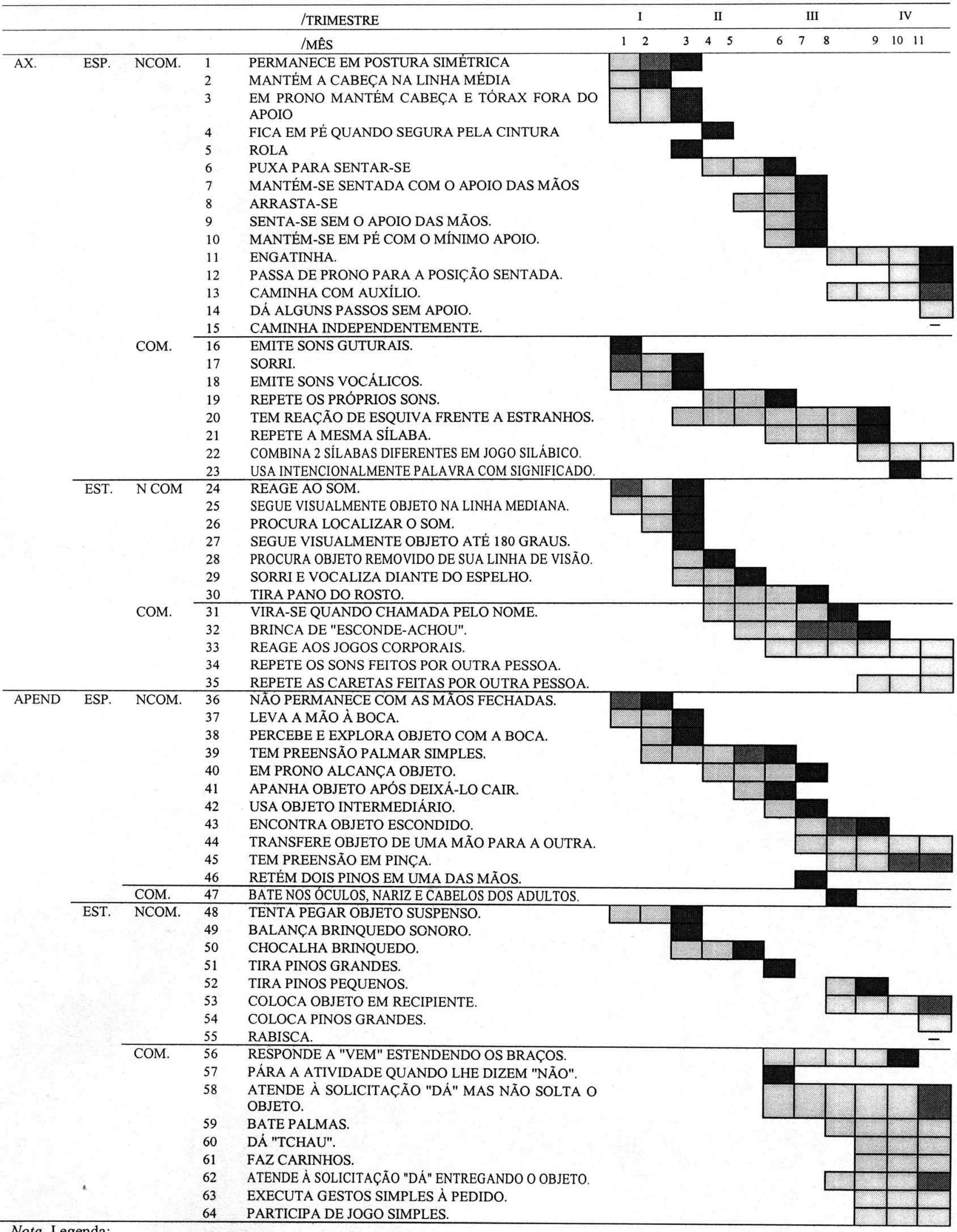


Os resultados deste estudo não podem ser diretamente comparados aos obtidos por Ramos (1999), pois apesar do instrumento utilizado ser o mesmo, foi considerada nesta investigação a faixa etária de um a doze meses, mais ampla do que o estudo anterior (Ramos, 1999), que foi de seis a doze meses, e a forma de análise foi diversa. Porém, em ambos os estudos foram encontradas algumas diferenças significantes no desenvolvimento do comportamento de crianças prematuras quando comparado ao de crianças nascidas a termo.

Os resultados também não podem ser diretamente comparados com o estudo de Linhares et al. (2003) sem que sejam consideradas as importantes diferenças das amostras estudadas, tendo em vista que as autoras referidas investigaram crianças com idade gestacional inferior a 34 semanas e peso igual ou inferior a $1500 \mathrm{~g}$ e a presente investigação incluiu a idade gestacional de 33 a 36 semanas. Além disso as análises realizadas por Linhares et al. (2003) consideraram a idade corrigida e nós optamos por uma análise geral sem a correção da idade e uma análise específica final com a idade corrigida. Assim, apesar dos diferentes delineamentos, os resultados dos dois estudos se aproximam e apontam na mesma direção, pois considerando o final do primeiro ano de vida, neste estudo obtivemos $23 \%$ de comportamentos de risco (não normalizados na idade), enquanto que Linhares et al. (2003), obtiveram $36 \%$ de comportamentos de risco com a idade corrigida. Ambos os estudos apontam para a importância do acompanhamento e da intervenção precoce diante dos problemas de desenvolvimento das crianças nascidas na condição de prematuridade.

Mesmo esta pesquisa tendo sido realizada com um grupo reduzido de crianças, espera-se ter contribuído para avanços no conhecimento sobre o desenvolvimento do comportamento do prematuro, abrindo-se possibilidades para outros estudos aprofundados na área. Ainda, como esta investigação concentrou-se no desenvolvimento do comportamento da crianca prematura até doze meses incompletos, e este desenvolvimento apresentou diferenças importantes quando comparado com o da criança nascida a termo, fazem-se necessários outros estudos, que posssam analisar como prossegue este desenvolvimento a partir dos 12 meses e, se as alterações verificadas, são superadas ou não com a idade.

Além disso, como um benefício adicional, a avaliação do bebê utilizando a Escala de Desenvolvimento do Comportamento da Criança-EDCC (Batista Pinto et al., 1997) na presença dos pais, pode ser uma forma preventiva de intervenção, tendo em vista que possibilita aos pais conhecer melhor as competências e características do comportamento do seu bebê, habilitando-os possivelmente a responder melhor às necessidades adaptativas da criança e a estabelecer com a ela uma interação mais satisfatória.

\section{Conclusão}

Esta investigação concluiu que, de forma geral, as crianças prematuras no primeiro ano de vida: (a) apresentaram uma freqüência relativa de comportamentos estabilizados, em ambos os sexos, maior do que a de comportamentos normalizados, indicando um risco importante no desenvolvimento de seus comportamentos; (b) obtiveram resultados equivalentes (em ambos os sexos) na normalização como na estabilização dos comportamentos axial, espontâneo, estimulado, não-comunicativo e comunicativo. O comportamento apendicular apresentou uma freqüência ligeiramente mais elevada do que os outros, tanto na normalização como na estabilização; (c) mostraram uma freqüência relativa de comportamentos normalizados no $1^{\circ}$ trimestre bem inferior aos outros trimestres, sendo a diferença estatisticamente significante para os comportamentos axial, apendicular, espontâneo, estimulado e não-comunicativo. O comportamento comunicativo não apresentou diferença significante entre os trimestres quanto à normalização; (d) apresentaram uma freqüência relativa de comportamentos estabilizados no $1^{\circ}$ trimestre também inferior aos outros trimestres, com diferença estatisticamente significante para os comportamentos axial, apendicular, estimulado, não-comunicativo e comunicativo. O comportamento espontâneo não apresentou diferença significante entre os trimestres quanto à estabilização; (e) mostraram uma boa recuperação no rítmo de desenvolvimento no período de 1 a 12 meses incompletos reduzindo, a partir do $3^{\circ}$ mês de vida, de forma estatisticamente significante, os comportamentos com indicadores patológicos, mas ainda assim mantendo um fator de risco de $23 \%$ de comportamentos não normalizados aos 12 meses incompletos.

A Escala de Desenvolvimento do Comportamento da Criança - EDCC (Batista Pinto et al., 1997) possibilitou aprofundar os conhecimentos sobre o processo de desenvolvimento do comportamento da criança prematura no primeiro ano de vida, em suas várias fases e características, permitindo uma análise qualitativa detalhada e favorecendo a detecção de atrasos e problemas no desenvolvimento do comportamento.

Desta forma a Escala de Desenvolvimento do Comportamento da Criança - EDCC (Batista Pinto et al., 1997) pode contribuir tanto preventivamente como interventivamente, possibilitando que pais e profissionais conheçam detalhadamente as competências e características do comportamento do bebê prematuro no primeiro ano de vida, e se tornem mais habilitados para responder às suas necessidades adaptativas e de estimulação.

\section{Referências}

Agência Nacional de Saúde Suplementar. (2005). Taxa de prematuridade. Brasília, DF: Autor. Retrieved September 20, 2005, from http://www.ans.gov.br/portal/site/-qualificação/pdf/ atenc_saude1fase.pdf

Als, H. (1995). The preterm infant: A model for the study of fetal brain expectation. In J.-P. Lecanuet, W. P. Fifer, N. A. Krasnegor, \& W. P. Smotherman (Eds.), Fetal development, a psychological perspective (pp. 439-472). Hillsdale, NJ: Laurence Erlbaum. 
Wiese, E. B. P. (2009). O Desenvolvimento do Comportamento do Bebê Prematuro no Primeiro Ano de Vida.

Atkin, L. C., Supervielle, T., Sawyer, R., \& Cantón, P. (1987). Paso a paso. Cómo evaluar el crecimiento y desarrollo de los niños. Mexico, DF: Pax Mexico.

Batista Pinto, E. (2000). La psychothérapie mère/bébé: un abordage de l'enfant prématuré. Symposium conducted at the meeting of the World Association of Infant Mental Health Congress - WAIMH, Montréal, Canada.

Batista Pinto, E., Vilanova, L. C. P., \& Vieira, R. M. (1997). $O$ desenvolvimento do comportamento da criança no primeiro ano de vida: Padronização de uma escala para a avaliação $e$ o acompanhamento. São Paulo, SP: Fundação de Amparo à Pesquisa do Estado de São Paulo.

Barratt, M. S., Roach, M. A., \& Leavitt, L. A. (1996). The impact of low-risk prematurity on maternal behaviour and toddler outcomes. International Journal of Behavioral Development, 19(3), 581-602.

Bullinger, A., \& Goubet, N. (1999). Le bébé prématuré, acteur de son développement. Enfance, 1, 27-32.

Charavel, M. (2000). Évolution de l'attitude des mères d'enfant prématuré et des mères d'enfant à terme en interaction avec leur bébé: une étude éthologique de la naissance à 6 mois. $L a$ Psychiatrie de l'enfant, 43(1), 175-206.

Coll, C. T. G. (1990). Developmental outcome of minority infants: A process-oriented look into our beginnings. Child Development, 61, 270-289.

Dalla Piazza, S. (1997). L'enfant prématuré: le point sur la question. Bruxelles, Belgique: De Boeck Université.

François, A., Battisti, O., Bertrand, J. M., Kalenga, P., \& Langhendries, J. P. (1998). Bébé prématuré, bébé particulier? Quel suivi développemental? Archives de Pédiatrie, 5, 568572.

Guralnick, M. J. (1998). Effectiveness of early intervention for vulnerable children: A development perspective. American Journal on Mental Retardation, 102(4), 319-345.

Lequien, P. (1999). Survie et qualité de survie des grands prématurés. Enfance, 1, 93-95.

Linhares, M. B. M., Carvalho, A. E. V., Bordin, M. B. M., \& Jorge, S. M. (1999). Suporte psicológico ao desenvolvimento de bebês prematuros com peso de nascimento de $<1500 \mathrm{~g}$ : Na UTI Neonatal e no seguimento longitudinal. Temas de Psicologia da SBP, 8(3), 162-245.

Linhares, M. B. M., Carvalho, A. E. V., Machado, C., \& Martinez, F. E. (2003). Desenvolvimento de bebês nascidos pré-termo no primeiro ano de vida. Cadernos de Psicologia e Educação Paidéia, 13(25), 59-72.

Mellier, D. (1999). La prématurité: l'ouverture de problématiques nouvelles. Enfance, 1, 3-11.

O'Brien, M., Soliday, E., \& McCluskey-Fawcett, K. (1995). Prematurity and the neonatal intensive care unit. In M. C. Roberts, Handbook of pediatric psychology (pp. 463-478). New York: Guilford.

Organização Mundial de Saúde. (1980). The incidence of low birth weight: A critical review of available information. World Health Statistics Quarterly, 33, 197-224.

Ortiz, E. R. (1987). O bebê de alto risco neurológico, o conceito de risco. In J. R. S. Lippi \& A. R. Cruz (Eds.), Neurologia infantil - Estudo multidisciplinar (pp. 27-34). Belo Horizonte, MG: ABENEPI.

Padovani, F. H. P., Linhares, M. B. M., Carvalho, A. E. V., Duarte, G., \& Martinez, F. E. (2004). Avaliação de sintomas de ansiedade e depressão em mães de neonatos pré-termo durante a após hospitalização em UTI-neonatal. Revista Brasileira de Psiquiatria, 26(4), 251-254.
Pérez-Ramos, A. M. Q., Pérez-Ramos, J., Sá, T. M. P., Morais, S. T. P., \& Jaehn, S. M. (1992). Estimulação precoce: Serviços, programas e currículos. Brasília, DF: Ministério da Ação Social.

Ramos, M. I. B. (1999). Comparação do comportamento de crianças pré-termo e a termo de 6 a 12 meses de idade. Monografia de Especialização não-publicada, Universidade Federal de São Paulo, SP.

Siegel, S. (1975). Estadistica no parametrico. Mexico, DF: Trillas.

Thoman, E. B., \& Ingersoll, E. W. (1993). Learning in premature infants. Developmental Psychology, 29(4), 692-700.

Thoman, E. B., Ingersoll, E. W., \& Acebo, C. (1991). Premature infants seek rhythmic stimulation, and the experience facilitates neurobehavioral development. Developmental and Behavior Pediatrics, 12(1), 11-18.

Tremblay-Leveau, H., Lemaitre-Boquet, L., Megan, A., Renard, S., \& Delaunay, H. (1999). Les actions de communication chez les bébés prématurés. Enfance, 1, 33-42.

Victora, C. G., Barros, F. C., Martines, J. C., Béria, J. U., \& Vaughan, J. P. (1985). Estudo longitudinal das crianças nascidas em Pelotas, RS, Brasil: Metodologia e resultados preliminares. Revista de Saúde Pública, 19, 58-68.

Widerstrom, A. H. (1999). Identification and treatment of risk factors in newborns and infants in the United States. Enfance, 1, 79-91.
Recebido: 01/05/2007

$1^{a}$ revisão: $24 / 03 / 2008$ Aceite final: 03/07/2008 